Piotr Politański ${ }^{1}$

Halina Aniołczyk ${ }^{1}$

Elżbieta Gadzicka ${ }^{2}$

Alicja Bortkiewicz ${ }^{2}$

Marek Zmyślony ${ }^{1}$

\title{
ELECTROMAGNETIC FIELDS EXPOSURE ASSESSMENT AMONG WORKERS AT BROADCAST CENTERS IN POLAND
}

Nofer Institute of Occupational Medicine, Łódź, Poland

${ }^{1}$ Department of Radiological Protection

${ }^{2}$ Department of Work Physiology and Ergonomics

\begin{abstract}
Background: In the studies investigating the potential health effects of occupational exposure to electromagnetic fields (EMF), the key problem is the assessment of workers' exposure. Electromagnetic fields exposure assessment requires determining maximum EMF levels, for the acute effect, and the dose absorbed, for the cumulative effect. A study was undertaken to determine EMF exposure in workers at broadcasting centers (BCs) in Poland. It was the first part of a comprehensive project on health effects of occupational exposure to EMF. Material and Methods: The study was conducted in 4 selected BCs. Spot measurements of electric field strength (E), and the results of E measurements that were made in the past for occupational hygiene assessments, were used for determining workers' exposure to electric field. The parameters considered were the maximum $E$ value $\left(\mathrm{E}_{\max }\right)$, the weighted average $\mathrm{E}$ value $\left(\mathrm{E}_{\text {avg }}\right)$ and the $\mathrm{E}$ lifetime dose $\left(\mathrm{E}_{\text {lifetime dose }}\right)$. Results: In the group of workers examined, the $\mathrm{E}$ values did not exceed $16.7 \mathrm{~V} / \mathrm{m}$ for $\mathrm{E}_{\max }$ (the occupational exposure limit (OEL) for work shift in Poland is $20 \mathrm{~V} / \mathrm{m}$ ), $2.9 \mathrm{~V} / \mathrm{m}$ for $\mathrm{E}_{\text {avg }}$ and $1500000(\mathrm{~V} / \mathrm{m})^{2} \times \mathrm{h}$ for $\mathrm{E}_{\text {lifetime dose. }}$ Conclusions: In view of the lack of dosimetry data, a retrospective assessment of BC workers' exposure to very high frequency and ultra-high frequency EMF was conducted, which made it possible to estimate EMF lifetime dose for the workers. Since the EMF exposure levels at BCs did not exceed the admissible values, they can be considered safe to the workers' health, according to the approach used for developing OELs for EMF exposure. Med Pr 2018;69(5):477-482
\end{abstract}

Key words: occupational exposure, television, electromagnetic fields, exposure assessment, radio, work safety in electromagnetic fields

Corresponding author: Piotr Politański, Nofer Institute of Occupational Medicine, Department of Radiological Protection, św. Teresy 8, 91-348 Łódź, Poland, e-mail: piotr.politanski@imp.lodz.pl

Received: August 24, 2017, accepted: February 20, 2018

\section{INTRODUCTION}

Radio and television are one of the fastest growing areas of human technological activities. Very wide-range-frequency electromagnetic field (EMF), varying from several dozen $\mathrm{kHz}$ to several $\mathrm{GHz}$ is used for broadcasting radio and television programs. The frequency bands include long-wave radiation $(30-300 \mathrm{kHz})$, medium frequency $(0.3-3 \mathrm{MHz})$, high frequency $(3-30 \mathrm{MHz})$, very high frequency (VHF) $(30-300 \mathrm{MHz})$ and ultra-high frequency (UHF) (0.3-3 GHz) EMF bands, with VHF and UHF bands being the major carriers of radio and television programs. To ensure that the programs are broadcast to as large country areas as possible, the broadcasting antennas are installed on freestanding masts or positioned on the roofs of tall buildings. One location is usually used for installing multiple antenna systems. This is how the radio and television broadcast- ing centers have emerged, that consist of several transmitters operating at different frequencies, and several antennas and feeders connecting the transmitters with the antennas. In Poland, in the 1960s-1970s, a network of broadcasting centers (BCs) with very tall masts, of over $200 \mathrm{~m}$ in height, was developed which incorporated most of the broadcasting stations and regional programs across the country. Today, the BCs, with their extensive infrastructure and workers' expertise, are commonly used by various broadcasters. The BC workers who had been exposed to VHF and UHF EMF for a long time are one of the occupational groups pointed out by Breckenkamp et al. [1] as a group which could be selected for a retrospective cohort study. Following a similar lead, authors undertook to the comprehensive project on health effects of occupational exposure to EMF sources in the BCs. There is definitely a need for this kind of project as there are several stud- 
ies addressing the problem of the health impact of EMF exposure from BCs on populations living in their vicinity [2-4], but barely a few studies on occupational exposure to EMF [5].

The health and safety regulations that were in force in Poland at the time this study was conducted, were based on the principle of the protection zones classified as intermediate, hazardous and dangerous [6]. When occupational hygiene assessment regarding EMF exposure was carried out, the VHF and UHF electric fields were considered as belonging to the same range. The rules of working in the protective zones for $15 \mathrm{MHz}$ to $3 \mathrm{GHz}$ are summarized in the Table 1 . However, in 2016, respective Polish regulations were harmonized with the Directive 2013/35/EU of the European Parliament and of the European Council of 26 June 2013 on the minimum health and safety requirements regarding the exposure of workers to the risks arising from electromagnetic fields [7]. According to the directive, the Action Levels (ALs), i.e., "operational levels established for the purpose of simplifying the process of demonstrating the compliance with relevant exposure limit values (mean values established on the basis of biophysical and biological considerations, in particular on the basis of scientifically well-established short-term and acute direct effects" are used for assessing workers' exposure. The minimum ALs indicated in the directive for exposure to electric field at VHF and UHF bands (electric field strength $\mathrm{rms}$ ) are as follows: $61 \mathrm{~V} / \mathrm{m}$ in the $10-400 \mathrm{MHz}$ frequency range and $3 \times 10^{-3} \mathrm{f}^{1 / 2} \mathrm{~V} / \mathrm{m}$ $(60-135 \mathrm{~V} / \mathrm{m})$ in the $400 \mathrm{MHz}-2 \mathrm{GHz}$ range, where $\mathrm{f}$ is the frequency expressed in hertz.

In the studies investigating the potential health effects of occupational exposure to EMF, the key problem is the assessment of workers' exposure. In this study (for BC workers) this assessment was further complicated by the fact that EMF with different operating frequencies had been used throughout the workers' life- time exposure and even the results of the measurements are not quite reliable as their level of uncertainty ranged $10-60 \%$ [8]. Moreover, since the nature of the biological effect of EMF has not been elucidated thus far, EMF exposure assessment required determining maximum EMF levels, for acute effect, and the dose absorbed, for cumulative effect.

\section{MATERIAL AND METHODS}

\section{Measurement locations}

The study was conducted in 4 selected BCs that were located over flat land areas in Poland. They were launched in 1960s. The assessment of workers' exposure to EMF in these centers is particularly complicated in the case of multi-program broadcasting which is characterized by variable occupational exposure conditions resulting from changes in the number and type of transmitters used throughout the period of exposure. Since the period of work history in these centers is often as long as several decades, the assessment of workers' exposure must consider these changes, and the related variable EMF exposure values, from the beginning of $\mathrm{BC}$ operation.

\section{History of EMF exposure}

It should be noted that our study was conducted at the time when radio and television programs were broadcast in the analog system; thus our estimation of EMF exposure was limited to that type of broadcasting equipment. During the service life of BC facilities, 4 basic types of broadcasting equipment were used, namely: VHF broadcast transmitters operating at frequencies $66-74 \mathrm{MHz}$ (International Radio and Television Organisation (Organisation Internationale de Radiodiffusion - OIRT) range), the so-called "old" VHF transmitters, VHF broadcast transmitters operating at frequencies 87.5-108 MHz (International Radio Consul-

Table 1. Electric field strength occupational exposure limits (for $15 \mathrm{MHz}-3 \mathrm{GHz}$ frequency band) that had been in force in Poland at the time of the measurements [6]

\begin{tabular}{lccc}
\hline Zone & $\begin{array}{c}\mathrm{E}_{\min } \\
{[\mathrm{V} / \mathrm{m}]}\end{array}$ & $\begin{array}{c}\mathrm{E}_{\max } \\
{[\mathrm{V} / \mathrm{m}]}\end{array}$ & Permissible time of exposure \\
\hline Safe & - & 6.7 & no limits \\
Intermediate & 6.7 & 20.0 & work shift \\
Hazardous & 20.0 & 200.0 & $3200 / \mathrm{E}^{2}[\mathrm{~h} / 24 \mathrm{~h}]$ \\
Dangerous & 200.0 & - & exposure not allowed
\end{tabular}

$\mathrm{E}_{\min }-$ minimum electric field strength, $\mathrm{E}_{\max }-$ maximum electric field strength. 
tative Committee (Comité Consultatif International pour la Radio - CCIR) range), the so-called "new" VHF transmitters, TV transmitters operating at the I-III OIRT television frequency range $(49.75-223.25 \mathrm{MHz}$ image carrier frequency, $56.25-229.75 \mathrm{MHz}$ audio carrier frequency) and TV transmitters operating at the IV-V TV CCIR and OIRT range $(471.25-783.25 \mathrm{MHz}$ image carrier frequency, 477.75-789.75 $\mathrm{MHz}$ audio carrier frequency).

The operational history of BCs comprises 3 main periods:

- the initial period, until mid-90s of the 20th century, in which "old" VHF transmitters, TV transmitters from the I-III range, and a few transmitters from the IV and V range were operated;

the transitional period, until the end of 1999, characterized by the operation of both "old" and "new" VHF transmitters and a large number of IV-V range TV transmitters;

the modern period, from the beginning of 2000 , when the "old" VHF transmitters ceased to be used. Therefore, one can assume that by mid 1990s, BC workers were exposed mainly to VHF EMF.

The radio and television transmitters installed at broadcasting centers that operate at VHF and UHF bands usually produce signals with a power of a few $\mathrm{kW}$, while the gain of their transmitting antennas is of the order of a few $\mathrm{dB}$. Therefore, one can expect that their contribution to external EMF is significant. Apart from the radio and television broadcasting equipment, the $\mathrm{BCs}$ also house radio- and telecommunication equipment (radio lines, radiotelephones, mobile base stations); however, due either to the nature of the emitted radiation (narrow, strictly directional beam of radio line antennas), or its low power (several dozen watts at maximum), it is not likely that they have a significant contribution to workers' exposure to EMF. Besides, most of these devices are maintenance-free and, therefore, in this study, the analysis of workers exposure has been limited to the VHF and UHF bands.

Broadcasting centers workers may be exposed to EMF emitted by all the 3 major components of the broadcasting system: the transmitter, the feed line and the antenna. The nature of the EMF involved varies, depending on the type of job and the source of the field. All of the staff are exposed to EMF in the far zone of antenna radiation, while the persons responsible for the control and maintenance of the devices are also exposed to EMF prevailing in the near zone. As the transmitting devices used in the broadcasting centers are mainly electrical in nature, the magnetic EMF component (in both zones) is negligibly low in relation to the electric component. Therefore, it seems reasonable that any study on the biological and health effects of EMF among BC workers should be limited to the effects of exposure to the electric field.

\section{Methodology of EMF exposure assessment}

Since the history of EMF exposure at BCs is often complicated, exposure assessment for workers with a long duration of work at a given facility could not be based solely on single measurements. Therefore, we decided to adopt a retrospective approach, assuming that the spatial distribution of EMF in the facility was similar because of the similar wavelengths emitted by the transmitting antennas throughout the total period of their operation. On the other hand, the spectral composition and EMF values were subject to changes during this time period. Accordingly, the following dataset was used for carrying out a retrospective assessment:

results of spot measurements of electric field strength at a given facility, indicating recent exposure levels (recent maximum value and recent dose); results of electric field measurements that were conducted during routine work hygiene assessments conducted in the past. These were used for a retrospective assessment of the maximum electric field strength during occupational exposure;

information on the spectral composition of EMF at different stages of $\mathrm{BC}$ operation;

information about the technical parameters of the transmitting antennas installed in BCs at different stages of their operation.

In practice, the procedure for assessment of workers exposure was as follows:

1. From the total period of operation of a given $\mathrm{BC}$, the time periods of constant exposure (for each EMF band) were distinguished.

2. Measurements for assessment of recent exposure were performed.

3. Results of maximum electric field strength measurements were analyzed in order to calculate conversion coefficients and estimate the changes in exposure level over the lifetime of a given $\mathrm{BC}$ (based on data from work hygiene protocols for EMF measurements performed previously in the facility).

4. The data referred to at point 2 were converted by using coefficients from point 3 for the time periods of constant exposure distinguished at point 1 . 
The assessment of EMF doses received by BC workers was made based on quasi-dosimetric measurements (as reliable electric field dosimeters were not available) and required estimation of the time the workers spent in particular areas of the facility with different EMF levels and determination of the average electric field intensity in these areas. In practice, a series of spot measurements at several locations were made in areas where the workers stayed during the work shift, and the results were the time-weighted average for a given area.

In the case the data was below the sensitivity level of the probe, the weighted average was calculated based on "technical specifications for chemical analysis and monitoring of water status" provided in the EC Directive 2009/90/EC of 31 July 2009 laying down, pursuant to the Directive 2000/60/EC of the European Parliament and of the European Council [9]. According to Article 5 of the Directive:

Where the amounts of physico-chemical or chemical measurements in a given sample are below the limit of quantification, the measurement results shall be set to a half of the value of the limit of quantification concerned for the calculation of mean values.

Where a calculated mean value of the measurement results referred to in paragraph 1 is below the limits of quantification, the value shall be referred to as less than the limit of quantification.

This estimation was made for about $40 \%$ of the measurement spots at BCs for which the measured values appeared to be lower than the probe sensitivity. Also, the maximum value of electric field strength to which the workers could have been exposed was determined for each study area of the facility. This data allowed to determine workers' exposure in terms of: maximum $E$ value $\left(E_{\text {max }}\right)$, weighted average $E$ value $\left(E_{\text {avg }}\right)$ and lifetime dose of exposure to electric field ( $\left.\mathrm{E}_{\text {lifetime dose }}\right)$.

$$
\mathrm{E}_{\text {lifetime dose }}=\left(\mathrm{E}_{\text {avg }}\right)^{2} \times \mathrm{t} \times \mathrm{T} \times \mathrm{N}
$$

where:

$\mathrm{t}$ - the duration of work shift (h),

$\mathrm{T}$ - the number of workdays per year,

$\mathrm{N}$ - the number of years of work at a given facility.

Since the main sources of EMF in BCs are the transmitting devices operating at VHF and UHF bands, the assessment was carried out separately for each type, as well as for the superposition of these fields (which is the square root of the sum of the squares of these fields). For the purpose of this study, the microwave power densi- ty values from work hygiene protocols for EMF measurements at the facilities operating at UHF band were converted to the electric field strength, using the relationship for plane wave. The exposure assessment was analyzed for the 3 different periods of BC operational history: the initial period (up to mid 1990s), the transitional period (to the end of 1999), and the contemporary period, with all data pooled into transversal analysis. An accurate evaluation of EMF exposure was necessary for a further assessment of exposure-related health effects (stage II of the project, reported elsewhere).

\section{EMF measuring set}

For EMF measurements, the MEH-25 measuring set developed at the Technical University of Wrocław, Poland, was used. The specific equipment included:

aE-2 probe (frequency range $10-300 \mathrm{MHz}$ ), measuring range: $0.4-15 \mathrm{~V} / \mathrm{m}$ ( $\mathrm{rms})$, with inaccuracy of EMF measurements in the free space $\pm 10 \%$ and $\pm 3 \mathrm{~dB}$ for measurements at $10 \mathrm{~cm}$ distance from primary and secondary radiation sources;

AS-2 probe (frequency range $0.4-14 \mathrm{GHz}$ ), measuring range: $0.005-100 \mathrm{~W} / \mathrm{m}^{2}$, with inaccuracy of EMF measurements in the free space $\pm 15 \%$ and $\pm 4 \mathrm{~dB}$ for measurements at $10 \mathrm{~cm}$ distance from primary and secondary radiation sources.

As shown above, EMF frequency ranges measured by the probes did not overlap (the upper limit of the frequency range for $\mathrm{AE}-2$ probe is $300 \mathrm{MHz}$, while the lower limit for AS-2 probe is $400 \mathrm{MHz}$ ). The measurement error due to the impact of EMF at frequencies outside the measuring range is about $10 \%$; thus it had no major impact on the quality of the results.

\section{Statistical analysis}

As the collected data had a log-normal distribution, they were averaged geometrically for the purpose of this analysis.

\section{RESULTS AND DISCUSSION}

Workers' exposure to VHF and UHF EMF at 4 selected BCs was assessed based on the described above history of exposure in particular facilities. The results of this evaluation, including the maximum/mean values for electric field strength measured in a given facility and the lifetime dose received by the workers, are shown in the Table 2. This assessment was made for all workers, regardless of their tasks. The magnitude of EMF exposure differed for the technical staff 


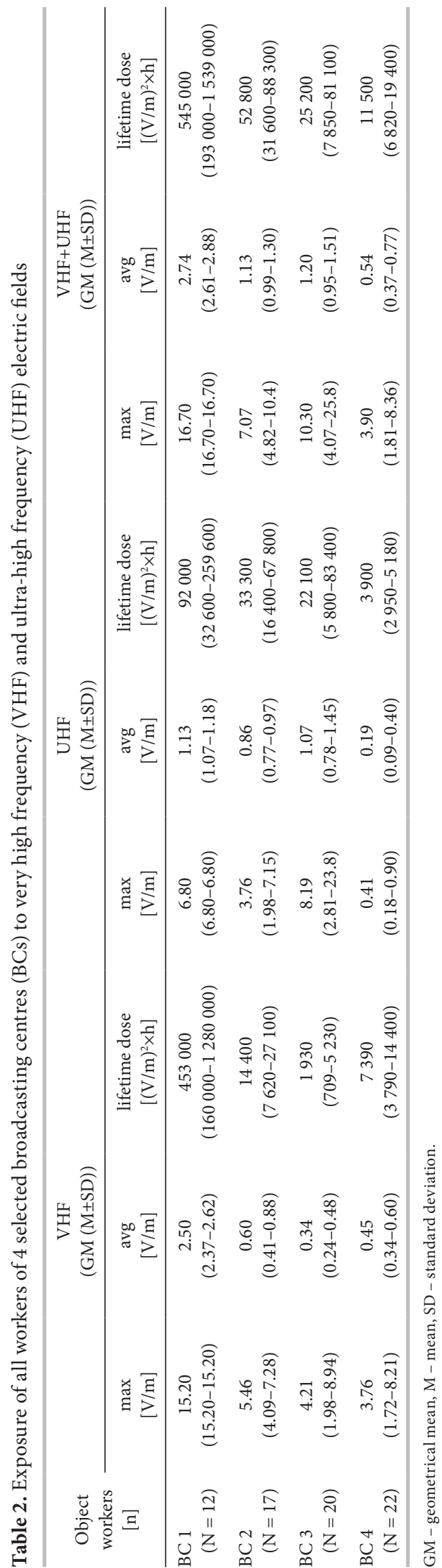

(managers and operators), the auxiliary staff (administration, gardeners, drivers, etc.) and the security staff, mostly owing to their different tasks and working time spent in particular areas of the facility. For example, the auxiliary and security staff did not stay in the vicinity of the transmitters, and thus they were exposed only to EMF emitted by the antennas (and, to a much lesser extent, by feedlines). Therefore, exposure assessment was conducted individually for each type of work.

The findings in the Table 2 show that BC workers were exposed mainly to VHF EMF. However, when the operation of the "old" VHF transmitters had been discontinued, the contribution of UHF EMF to the total spectrum became more significant. To determine whether exposure to both EMF bands emitted at BCs (Table 2) is high, it was compared with the OEL values that had been in force in Poland at the time of the measurements (Table 1).

The comparison of data shown in the Table 2 with the normative values in the Table 1 , indicates that workers at the $\mathrm{BC}$ facilities under study were exposed to VHF and UHF EMF, with their maximum levels corresponding to intermediate zone conditions, and that their exposure did not seem to produce any adverse health effects according to the approach used for developing OELs for EMF exposure. If one were to take into account the exposure index (the ratio of effective dose to dose limit $\left.\left(3200 \mathrm{~V}^{2} \mathrm{~h} / \mathrm{m}^{2}\right)\right)$, the workers' exposure at BCs might be considered negligible (the exposure index ranged from $1.6 \times 10^{-6}$ to $1.3 \times 10^{-2}$ ), depending on the period of operation and a given facility. The relatively low impact of the exposure was also confirmed by the fact that EMF doses received by BC workers during 24-h exposure never exceeded the daily doses specified in the legal regulations on the health protection for humans and the environment [10]. For example, for the general public, the admissible daily dose for EMF at VHF band is $96 \mathrm{~V}^{2} \mathrm{~h} / \mathrm{m}^{2}$, while in our study, the maximum daily dose for BC workers was about $38 \mathrm{~V}^{2} \mathrm{~h} / \mathrm{m}^{2}$.

Besides, comparing these values with the ALs specified in the EC directive revealed that the $\mathrm{E}_{\max }$ levels (of less than $20 \mathrm{~V} / \mathrm{m}$ ) to which the BC workers were exposed to were much lower than the corresponding ALs. Thus one can conclude that this exposure is not likely to produce any harmful effects to human health, according to the provisions of the Directive.

There is very limited data on occupational exposure to EMF at BCs. The findings of our study are consistent with other reported data from Poland [11], where the values ranging $0.4-9.6 \mathrm{~V} / \mathrm{m}$ were found in workplac- 
es operating at VHF (87.5-108 MHz). Electromagnetic fields exposure in Polish BCs was slightly lower than that reported by Vangelova et al. in the Bulgarian Broadcast Centers [5], where the equivalent time-weighted average was $2.12-3.9 \mathrm{~V} / \mathrm{m}$ (vs. $0.54-2.74 \mathrm{~V} / \mathrm{m}$ in this study). The lower exposure level in Poland may be attributed to a better management of working under conditions of EMF exposure. As pointed out by Breckenkamp et al. [1], for the personnel of medium/short wave broadcasting stations "The limitations of the study design include the rather low levels of exposure (mechanical workshop, Mühlacker broadcasting station, electrical field: $1.5 \mathrm{~V} / \mathrm{m}$, magnetic field: $0.2 \mathrm{~A} / \mathrm{m}$ ), the small cohort size ( $\max .250$ potentially exposed persons in Germany) and the fact that such a cohort consists exclusively of persons in technical occupations (highly selective group)." Therefore, we decided to assess exposure not only for the technical occupations but also for the auxiliary personnel and security staff, and the levels of exposure we recorded were similar to those reported by Breckenkamp et al. [1].

\section{CONCLUSIONS}

In view of the lack of dosimetry data, a retrospective assessment of BC workers' exposure to VHF and UHF EMF was conducted, which made it possible to estimate the lifetime EMF dose for the workers. To our knowledge, this has been the first attempt of this kind.

As the EMF exposure levels at BCs did not exceed the admissible values, they can be considered safe to the workers' health, according to the approach used for developing occupational exposure limits for EMF exposure.

\section{REFERENCES}

1. Breckenkamp J, Berg-Beckhoff G, Münster E, Schüz J, Schlehofer B, Wahrendorf J, et al. Feasibility of a cohort study on health risks caused by occupational exposure to radiofrequency electromagnetic fields. Environ Health. 2009;8:23, https://doi.org/10.1186/1476-069X-8-23.

2. Gajšek P, Ravazzani P, Wiart J, Grellier J, Samaras T, Thuróczy G. Electromagnetic field exposure assessment in Europe radiofrequency fields (10 MHz-6 GHz). J Expo Sci Environ Epidemiol. 2015;25(1):37-44, https://doi.org/10.1038/ jes.2013.40.
3. Hauri DD, Spycher B, Huss A, Zimmermann F, Grotzer M, von der Weid N, et al. Exposure to radio-frequency electromagnetic fields from broadcast transmitters and risk of childhood cancer: A census-based cohort study. Am J Epidemiol. 2014;179(7):843-51, https://doi.org/10.1093/aje/ kwt442.

4. Schmiedel S, Brüggemeyer H, Philipp J, Wendler J, Merzenich $\mathrm{H}, \mathrm{Schüz} J$. An evaluation of exposure metrics in an epidemiologic study on radio and television broadcast transmitters and the risk of childhood leukemia. Bioelectromagnetics. 2009;30(2):81-91, https://doi.org/10.1002/ bem. 20460 .

5. Vangelova K, Deyanov C, Israel M. Cardiovascular risk in operators under radiofrequency electromagnetic radiation. Int J Hyg Environ Health. 2006;209(2):133-8, https://doi. org/10.1016/j.ijheh.2005.09.008.

6. [Regulation of the Minister for Labour and Social Policy of 2 January 2001 amending the regulation on the maximum admissible concentrations and intensities of workplace hazards. J Laws 2001, item 36]. Polish.

7. Directive 2013/35/EU of the European Parliament and of the Council of 26 June 2013 on the minimum health and safety requirements regarding the exposure of workers to the risks arising from physical agents (electromagnetic fields) and repealing Directive 2004/40/EC. Off J Eur Union L 179 (Jun 29, 2013), https://doi.org/10.3000/19770677.L_2013. 179.eng.

8. Bieńkowski P. [Electromagnetic fields emitted in radio- and microwave- frequency range: Equipment and methods for the environment protection and survey measurements]. Med Pr. 2008;59(6):513-9.

9. Commission Directive 2009/90/EC of 31 July 2009 laying down, pursuant to Directive 2000/60/EC of the European Parliament and of the Council, technical specifications for chemical analysis and monitoring of water status (Text with EEA relevance). Off J Eur Union L 201/36 (Aug 1, 2009).

10. [Regulation of the Minister for Environment of 30 October 2003 on the permissible levels of electromagnetic fields in the environment and ways to check compliance with these levels. J Laws 2003, item 1883]. Polish.

11. Aniołczyk H, Mariańska M, Mamrot P. [Assessment of occupational exposure to radio-frequency electromagnetic fields]. Med Pr. 2015;66(2):199-212, https://doi.org/10.13 075/mp.5893.00196. Polish.

This work is available in Open Access model and licensed under a Creative Commons Attribution-NonCommercial 3.0 Poland License - http://creativecommons.org/licenses/by-nc/3.0/pl/deed.en. 